\title{
PERCEPCIÓN DEL CLIMA SOCIAL LABORAL Y DE LA EFICIENCIA PERSONAL EN PROFESIONALES DE LA SALUD DEL SECTOR PÚBLICO DE LA CIUDAD DE LIMA
}

\author{
PERCEPTION OF THE LABOR SOCIAL CLIMATE AND THE PERSONAL \\ EFFICIENCY IN PROFESSIONALS OF THE HEALTH OF THE PUBLIC SECTOR OF \\ THE CITY OF LIMA
}

\author{
MARIO SANTIAGO BULNES BEDÓN ${ }^{1}$ CARLOS RAMON PONCE DIAZ, ROSA \\ ELENA HUERTA ROSALES, ROGER ELIZAL DE BRAVO, WILLY RE NATO \\ SANTIVÁÑEZ OLULO, ELIANA DELGADO COZ, LENI ALVAREZ TACO \\ Universidad Nacional Mayor de San Marcos, Facultad de Psicología
}

\begin{abstract}
RESUMEN
OBJETIVOS: Precisar la interacción entre la Percepción del Clima Social Laboral y la Percepción de la eficiencia personal en los profesionales de la salud. DISEÑO: Es descriptivo, comparativo y correlacional, de carácter analítico en la cual se trató de determinar la prevalencia y distribución de desgaste profesional en los profesionales de la salud. MATERIAL Y MÉTODOS: Está conformada por 93 profesionales de la salud de centros hospitalarios públicos entre médicos, enfermeras, obstetrices, psicólogos, trabajadores sociales y tecnólogos médicos. Para la evaluación de las variables se utilizaron La Escala de Clima Social Laboral elaborado por Rudolf Moos y Bernice Moos, y el Inventario de Burnout elaborado por Cristina Maslach y Susan Jackson. RESULTADOS:

Al estudiar las correlaciones de las variables percepción del clima social y percepción de la eficiencia personal, medidas por la escala de clima social laboral y la prueba de estrés laboral o burnout, respectivamente, se pudo observar que no existe una correlación significativa entre ambas variables. Tales resultados pueden ser interpretados en el sentido de tratarse de dos variables autónomas. Sin embargo, al interior de cada escala sí se observan correlaciones significativas, como es obvio se encuentran relacionadas con la construcción y estructuración de las mismas pruebas. CONCLUSIONES: No existe una correlación significativa entre las escalas de estrés laboral y la escala de clima social laboral en la muestra estudiada; no se observan diferencias significativas en ambas variables en función al género; desde el punto de vista del análisis factorial, las diez subescalas de la prueba de Clima Social Laboral pueden agruparse en dos componentes básicos.
\end{abstract}

Palabras claves: Atención de salud, desgaste profesional, Burnout, Cuestionario, Clima Laboral.

\begin{abstract}
OBJECTIVES: To need interaction between the Perception the Labor Social Climate and the Perception of the personal efficiency in the professionals of the health. DESIGN: He is descriptive, comparative and corelational, of charater analytic in which it treated to determine the prevalence and distribution of professional wearing down in the professionals of the health. MATERIAL AND MÉTODOS: It is conformed by 93 professionals of the health of publics hospitable centers between social doctors, nurses, obstetrics, psychologists, workers and technologies doctors. For evaluation of the variables the Climate Scale elaborated by Rudolf Moos and Bernice Moos, and the Inventory of Burnout. Were used Labor Social elaborated by Cristina Maslach and Susan Jackson.
\end{abstract}

${ }^{1}$ Email: mbulnesbedon@unmsm.edu.pe 
RESULTS: When study the correlations of the variables perception of the social el climate and perception of the personal efficiency, measured by the labor social elimate scale and the test of labor stress or burnout, respectively, could be observed that a significant correlation between both variables does not exist. Such results can be interpreted in the sense to be two independent variables. Nevertheless, the interior of each scale yes significant correlations are observed, as it is obvious are related to the construction and structuring of the same tests. CONCLUSIONS: A significant correlation between the scales of labor stress and the labor social climate scale does not exist in the studied sample; significant differences in both variables in function are not observed I generate; from the point of view of the factorial analysis, the ten subscales of the test of Labor Social Climate can be grouped in two basic components.

Keywords: Attention of health, wears away professional, Burnout, Questionnaire, Labor Climate.

\section{INTRODUCCIÓN}

En la presente investigación se plantea la importancia del estudio de la Percepción del Clima Social Laboral y de la eficacia personal en profesionales de la salud, como factores desencadenantes del estrés laboral, lo que a su vez repercute en la calidad de atención a los pacientes.

Estudios epidemiológicos han demostrado que existe una relación entre salud y trabajo, donde se considera que los factores laborales pueden causar o agravar problemas de salud o por el contrario, pueden también favorecer la salud de los trabajadores, en este sentido, consideramos que en los centros hospitalarios -en la medida que constituyen organizaciones laborales-, también se evidencia esta relación entre salud y trabajo.

El estudio del clima social en contextos laborales (como por ejemplo, las organizacionales de salud) involucra el análisis de variables tanto físicas como sociales que se encuentran insertas en el mismo. Al respecto, Moos ${ }^{(1)}$, desde la Ecología Social, centra el estudio de la conducta humana, abordándola desde diversas variables, como son: arquitectónica, político organizativas del ambiente, características de los habitantes del contexto, así como dimensiones psicosociales de grupo e institucionales. Por otro lado, el riesgo de aparición del Síndrome de Burnout está asociado básicamente a factores organizacionales. De tal manera que no es la estructura de la personalidad o las formas de conducta lo significativo, sino el clima y la cultura laboral del propio ámbito de trabajo. Distintos niveles de malestar psíquico pueden surgir tanto por las condiciones laborales, como por la organización del trabajo, desarrollando como consecuencia de ello los llamados sistemas defensivos o también ideologías defensivas, frente a las situaciones de riesgo. Esto nos hace pensar la relación que puede existir entre el clima social laboral y las consecuencias emocionales que puedan suscitar en los diferentes profesionales que lo integran. De alguna forma esto justifica el interés de estudiar ambas variables en el presente contexto.

Los estudios sobre el Síndrome de Burnout desarrollado en varios países y en el nuestro, como es el caso de Ana Delgado ${ }^{(2)}$ realizado en docentes de entidades escolares públicas, o como la investigación realizada por Carlos Ponce ${ }^{(3)}$ en docentes universitarios, confirman los efectos del estrés asistencial relacionado con el objeto y condiciones de trabajo. En estos últimos tiempos se están realizando líneas de investigación en relación al clima social y

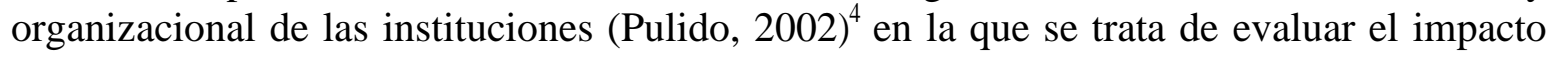
que puede tener dicho factor en la productividad, la eficiencia y calidad de los servicios. En este caso el propósito es indagar sobre las representaciones percibidos por los profesionales de la salud del sector público a través del Inventario del Clima Social Laboral de Moos, R. 
y Moos, $\mathrm{B}^{(1)}$

En 1975, Porter, Lawwer y Hackman (citado por Pulido, 2002) ${ }^{(4)}$ hablan de características concretas y prácticas habituales de la organización que van a ser percibidas por sus miembros o quienes estén familiarizados con el sistema.

De otro lado existen otros autores como Weinert $(1985)^{(5)}$, que analiza las tendencias del enfoque que se ha tenido para definir el concepto de Clima Organizacional. Él señala tres aspectos: el primero, que se refiere a las características objetivas de la organización circunscribiéndose a la realidad y a las dimensiones principalmente estructurales; el segundo aspecto, es la concepción del Clima Organizacional desde el punto de vista de la percepción individual de los atributos subjetivos y de la descripción, por ello se afirma que el Clima es una suma de variables que se constituye como una descripción de todos los estímulos que influyen en el individuo; y en tercer lugar encontramos al Clima Organizacional entendido como un clima psicológico que sería una cuestión puramente individual; .es decir, lo único importante es cómo se percibe el individuo mismo y como percibe su entorno laboral, sin tomar en consideración cómo se perciben otros en ese mismo entorno laboral. Por lo tanto, el Clima es una suma de percepciones con posibles secuelas comportamentales (Kolb, Rubin, Mclntyre, 1977) $)^{(6)}$ y es descriptivo de la organización global o de sus subsistemas. Como tal, Weinert ${ }^{(5)}$ refiere en opinión de Naylor(citado por Pulido) ${ }^{(4)}$ que dichos enfoques son partes de un proceso de desarrollo que parte de las características externas al individuo, pasa por los atributos subjetivos de la organización percibidos por el individuo, y llega al constructo psicológico de clima global izado y personalizado, el juicio descriptivo sobre la organización.

El Clima es, a decir de José María Peiró. (1984) ${ }^{(7)}$, percepciones, impresiones 〈〉imágenes de una realidad organizacional «subjetivada». La definición que se asevera en el presente análisis se sintetiza en una serie de características o atributos que distinguen una organización de otra. Así, Brunet (1987) se refiere al Clima como una medida perceptiva de los atributos organizacionales donde el individuo actúa como filtro de información de su ambiente ( $s u$ organización o sus subsistemas) así como de sus características personales, a través de las estructuras y procesos organizacionales. Las investigaciones sobre Síndrome de Burnout confirman los efectos del estrés asistencial relacionado con el objeto y condiciones de trabajo, sobre todo el personal médico, concentrando el mayor porcentaje del personal afectado, tanto médico como enfermera, la categoría de Despersonalización. Esto pone de manifiesto la relación que se establece con los pacientes, siendo indicativa de la sobrecarga que siente el profesional y el desarrollo de defensa como medida protectora. Estas defensas conlleva a la perturbación del vínculo terapéutico médico - paciente, enfermera - paciente y repercute en la calidad de la atención. Se ha podido ver en los médicos en los porcentajes de personal con más altos puntajes en los Servicios de Guardia, Obstetricia y Pediatría, lo que nos indica una vulnerabilidad en estas áreas.

Lo anteriormente mencionado conduce a buscar la relación entre las percepciones del Clima Social Laboral y la percepción de la eficiencia del profesional de la salud del Sector Público.

\section{Planteamiento del Problema}

El presente estudio pretende responder a las siguientes interrogantes:

1. ¿Cuál es la percepción del clima social laboral y la eficiencia profesional o estrés laboral en los profesionales de la salud?

2. ¿Cuál es la relación entre las variables clima social laboral y eficiencia laboral? 


\section{Objetivos}

1. Precisar la interacción entre la Percepción del Clima Social Laboral y la Percepción de la eficiencia personal en los profesionales de la salud

2. Conocer las características de las Percepciones del Clima Social Laboral en profesionales de la salud de especialidades diferenciadas.

3. Conocer las características de la percepción de la eficiencia personal en profesionales de la salud de especialidades diferenciadas.

4. Formular indicaciones con respecto a las dos variables y a la actividad de los profesionales de la salud.

\section{Justificación}

Permitirá conocer el Clima Social Laboral en el cual se desempeña el Profesional de la Salud del Sector Público. Se establecerá la relación que existe entre el Clima Social Laboral y la eficiencia profesional. Propiciar la interacción entre el Clima Social Laboral y la calidad de la atención de los profesionales de la salud. Fomentar la formulación de indicadores con respecto a la atmósfera laboral y la realización profesional.

\section{Hipótesis}

$\mathrm{H}_{1}$ : Existe una correlación entre la Percepción del Clima Social Laboral y la percepción de la eficiencia personal en los profesionales de la salud.

$\mathrm{H}_{2}$ : Existe diferencia entre los niveles de percepción del Clima Social Laboral y Percepción de la eficiencia personal en los profesionales de la salud considerando la situación laboral.

$\mathrm{H}_{3}$ : Existe diferencia entre los niveles de percepción del Clima Social Laboral y Percepción de la eficiencia personal en los profesionales de la salud considerando la condición de género.

$\mathrm{H}_{4}$ : Existe diferencia significativa entre los niveles de percepción del Clima Social Laboral y Percepción de la eficiencia personal en los profesionales de la salud considerando las especialidades.

$\mathrm{H}_{5}$ : Existe diferencia significativa entre los niveles de percepción del Clima Social Laboral y Percepción de la eficiencia personal en los profesionales de la salud considerando los tiempos de servicios.

\section{MATERIALES Y MÉTODOS.}

\section{Diseño de la Investigación}

Corresponde al descriptivo comparativo y correlacional, en el que se trata de describir y luego comparar por separado la percepción del clima social laboral y la percepción de la eficiencia personal en los profesionales de la salud de las entidades públicas. Luego se busca establecer la relación entre ambas variables motivo de estudio.

\section{Muestra}

Está conformada por 93 profesionales de la salud de centros hospitalarios públicos entre médicos, enfermeras, obstetrices, psicólogos, trabajadores sociales y tecnólogos médicos (técnico en rehabilitación, laboratorista, radiología) como se puede ver en el cuadro $\mathrm{N}^{\circ} 01$ que se detalla a continuación: 


\section{CUADRO $\mathbf{N}^{\circ} 01$ : Descripción de la muestra por especialidad}

\begin{tabular}{ccc}
\hline ESPECIALIDAD & $\boldsymbol{f}$ & $\boldsymbol{\%}$ \\
\hline MÉDICO & 14 & 15.1 \\
ENFERMERA & 17 & 18.3 \\
OBSTETRIZ & 16 & 17.2 \\
PSICÓLOGO & 10 & 10.7 \\
TRABAJO SOCIAL & 7 & 7.5 \\
TEC. MÉDICO & 29 & 31.2 \\
\hline TOTAL & $\mathbf{9 3}$ & $\mathbf{1 0 0 . 0}$
\end{tabular}

En el cuadro $\mathrm{N}^{\circ} 02$ se observa la muestra distribuida de acuerdo al tiempo de servicio, para lo cual se ha dividido en cuatro grupos de acuerdo a los cálculos realizados. El grupo "A" es el correspondiente a los que tienen de 1 a 8 años de servicio; el grupo "B" son los que tienen de 9 a 16 años de servicio; el grupo " $\mathrm{C}$ " son los profesionales que tienen entre 17 y 24 años de servicio; y por último, el grupo "D" son los profesionales que tienen entre 25 y 32 años de servicio.

\section{CUADRO $\mathrm{N}^{\circ}$ 02: Descripción de la muestra por tiempo de servicio}

\begin{tabular}{ccc}
\hline T. DE SERVICIO (en años) & $\boldsymbol{f}$ & $\boldsymbol{\%}$ \\
\hline Grupo "A" $1-8$ & 32 & 34.4 \\
Grupo "B" $9-16$ & 28 & 30.1 \\
Grupo "C" 17- 24 & 25 & $26: 9$ \\
Grupo "D" 25 - 32 & 8 & 8.6 \\
\hline TOTAL & 93 & 100.0
\end{tabular}

En el cuadro $\mathrm{N}^{\circ} 03$ se observa la muestra, motivo de estudio, dividido en dos grupos diferenciados por su condición laboral (contratados y nombrados), en una cantidad de 34 y 58 respectivamente.

\section{CUADRO N 03: Descripción de la muestra por situación laboral}

\begin{tabular}{ccc}
\hline Situación laboral & $\mathbf{f}$ & \% \\
\hline Contratado & 34 & 36.6 \\
Nombrado & 58 & 62.4 \\
\hline TOTAL & $\mathbf{9 3}$ & $\mathbf{1 0 0 . 0}$
\end{tabular}

En el cuadro $\mathrm{N}^{\circ} 04$, la muestra se halla dividido en función a sexo (masculino y femenino) en una cantidad de 26 y 67 respectivamente. 
CUADRO Nº4: Descripción de la muestra en función al sexo

\begin{tabular}{ccc}
\hline Sexo & $f$ & $\%$ \\
\hline Masculino & 26 & 28.0 \\
Femenino & 67 & 720 \\
\hline TOTAL & $\mathbf{9 3}$ & $\mathbf{1 0 0 . 0}$
\end{tabular}

\section{Variables de estudio}

Variable Independiente: Centros Hospitalarios, Especialidad profesional y tiempo de servicio.

Variable Dependiente: Los resultados derivados de las pruebas sobre Percepción del Clima Social Laboral y Percepción de la eficiencia personal (agotamiento profesional)

\section{Técnicas e instrumentos de recolección de datos}

Para la evaluación de las variables estudiadas se utilizaron los siguientes instrumentos psicológicos:

a. La Escala de (lima Social Laboral: El cual es un instrumento muy versátil elaborado por Rudolf Moos y Bernice Moos $\left.(1974)^{11}\right)$ llamado «La escala de ambiente de trabajo», aplicable a la mayoría de organizaciones en función a tres dimensiones y agrupadas en diez subescalas:

1. Relaciones Interpersonales: Es una dimensión que evalúa el grado en que los empleados están interesados y comprometidos en su trabajo y el grado en que la dirección apoya a los empleados y les anima a apoyarse unos a otros.

- IM Implicación (grado de compromiso de los empleados con su trabajo).

- $\boldsymbol{C O}$ Cohesión (relaciones de amistad y apoyo entre los trabajadores).

- AP Apoyo (apoyo y estímulo de la dirección para sus empleados).

2. Autorrealización: Evalúa el grado en que se estimula a los empleados a ser autosuficientes y a tomar sus propias decisiones; la importancia que se da a la buena planificación, eficiencia y culminación de las tareas y el grado en que la presión en el trabajo o la urgencia dominan el ambiente laboral.

- $\boldsymbol{A} \boldsymbol{U}$ Autonomía (grado en que la organización alienta la autonomía y toma de decisiones).

- OR Organización (grado en que el clima estimula la planificación y eficacia del trabajo).

- PR Presión (presión ejercida por la dirección para el cumplimiento del trabajo).

$\bullet$

3. Estabilidad y Cambio: Estas subescalas evalúan el grado en que los empleados conocen lo que se espera de su tarea diaria y cómo se les explican las normas y planes de trabajo; el grado en que la dirección utiliza las normas y la presión para controlar a los empleados; la importancia que se da a la variedad, el cambio y a las nuevas propuestas; también al grado en que el entorno físico contribuye a crear un ambiente de trabajo agradable. 
- $\boldsymbol{C L}$ Claridad (grado de claridad de reglamentos y políticas).

- $\boldsymbol{C N}$ Control (reglamentos y formas de presión que puede utilizar la dirección).

- IN Innovación (importancia que otorga la dirección al cambio y nuevas formas de trabajar).

- $\boldsymbol{C F}$ Comodidad (esfuerzos que realiza la dirección para crear un ambiente físico sano y agradable).

b. Inventario de "Burnout": Elaborado por Cristina Maslach y Susan Jackson, fue presentado en el Congreso Anual de la Asociación de Psicología (APA 1976) ${ }^{(9)}$, revisado y publicado por TEA Ediciones (1997). Es un instrumento que evalúa tres variables fundamentales del desgaste profesional :

1. CE Cansancio emocional: Constituida por 09 items, valora los sentimientos de una persona emocional mente saturada por el propio trabajo. Caracterizado por la pérdida progresiva de energía, de recursos personales de adaptación, desgaste y agotamiento.

2. DP Despersonalización: Constituida por 05 items. Mide el grado en la cual la respuesta hacia los pacientes es fría, distante e impersonal, manifestada por un cambio negativo de actitudes que lleva defensivamente a adoptar un distanciamiento frente a los problemas e incluso a proyectar el conflicto culpando a los propios pacientes de los problemas que le pasan al mismo profesional. Hay un camino progresivo a la "insensibilización» que $v a$ desde el pesimismo al cinismo respecto de los atendidos.

3. RP Falta de autorrealización personal:

Consta de 08 items y evalúa los sentimientos de competencia y eficacia en la realización del trabajo, donde se dan respuestas negativas hacia sí mismo y hacia el trabajo en un marco de fuerte insatisfacción interna, baja autoestima y fuertes autocuestionamientos, con manifestaciones pseudodepresivas y con tendencia a la huida.

\section{Técnicas de Procesamiento y Análisis de Datos}

a. Análisis descriptivo y exploratorio: frecuencia, porcentajes, media, desviación standard.

b. El análisis inferencial para la contrastación de hipótesis.

c. Análisis psicométrico de las Pruebas: Análisis de ítems con el cómputo de las correlaciones item-test, y la confiabilidad, cálculo de los coeficientes Alfa de Crombach.

\section{RESULTADOS}

Los resultados serán presentados de acuerdo a la siguiente secuencia:

A. Análisis de correlación de las variables motivo de estudio.

B. Análisis comparativo en función a la situación laboral.

C. Análisis comparativo en función al género.

D. Análisis comparativo en función a actividades profesionales diferenciadas.

E. Análisis comparativo en función al tiempo de servicio.

F. Análisis factorial de la prueba de clima social laboral.

Análisis de correlación de las variables motivo de estudio. Al estudiar las correlaciones de las variables percepción del clima social y percepción de la eficiencia personal, medidas por la escala de clima social laboral y la prueba de estrés laboral o burnout, respectivamente, se pudo observar que no existe una correlación significativa entre ambas variables. Tales resultados pueden ser interpretados en el sentido de tratarse de dos variables autónomas y por lo tanto no interdependientes. Sin embargo, al interior de cada 
escala sí se observan correlaciones significativas, como es obvio se encuentran relacionadas con la construcción y estructuración de las mismas pruebas.

CUADRO $\mathrm{N}^{\circ} 05$ : Correlación entre la prueba de estrés laboral (burnout) y clima social laboral

\begin{tabular}{|c|c|c|c|c|c|c|c|c|c|c|c|c|c|}
\hline & CEI & DPI & RPI & IM2 & $\mathrm{CO} 2$ & $\mathrm{AP2}$ & $\mathrm{AU2}$ & $\mathrm{OR}_{2}$ & PR2 & $\mathrm{CL} 2$ & $\mathrm{CN} 2$ & IN2 & $\mathrm{CF} 2$ \\
\hline CEI & I & 0.6106 & 0.3673 & 5 & - & - & 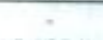 & 0.0538 & 0.2435 & - & 0.2416 & - & - \\
\hline & & & & 0.0196 & 0.0987 & 0.1209 & 0.1384 & & & 0.0892 & & 0.2285 & 0.0536 \\
\hline DPI & 0.6106 & 1 & 0.5116 & - & 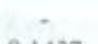 & + & - & 0.0134 & 0.0237 & - & 0.1345 & 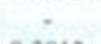 & - \\
\hline & & & & 0.0268 & 0.1437 & 0.1449 & 0.0564 & & & 0.1408 & & 0.2012 & 0.1509 \\
\hline RPI & 0.3673 & 0.5116 & 1 & - & - & - & - & + & 0.0417 & $*$ & 0.1284 & - & - \\
\hline & & & & 0.1923 & 0.2659 & 0.2118 & 0.2763 & 0.0974 & & 0.1815 & & 0.3667 & 0.1591 \\
\hline IM2 & a & - & - & 1 & 0.683 & 0.5504 & 0.5383 & 0.7388 & a isso & 0.6918 & 0.3363 & 0.6268 & 0.4526 \\
\hline $\mathrm{CO}_{2}$ & 0.0196 & 0.0268 & 0.1923 & & & & 0.628 & & $\begin{array}{c}0.1659 \\
.\end{array}$ & & & & \\
\hline & 0.0987 & 0.1437 & 0.2659 & 0.683 & 1 & 0.5912 & & 0.5302 & 0.2162 & 0.6. & 0.1 & 6 & 1 \\
\hline $\mathrm{AP} 2$ & 0,1209 & 0.1449 & 0.2118 & 0.5504 & 0.5912 & 1 & 0.6991 & 0.4736 & 0.1354 & 0.7161 & 0.0881 & 0.6007 & 0.3579 \\
\hline $\mathrm{AU} 2$ & 0.1384 & 0.0564 & 0.2763 & 0.5383 & 0.628 & 0.6991 & 1 & 0.472 & 0.2138 & 0.6863 & 0.0378 & 0.6082 & 0.2524 \\
\hline OR2 & 0.0538 & 0.0134 & 0.0974 & 0.7388 & 0.5502 & 0.4736 & 0.472 & 1 & 0.0585 & 0.6454 & 0.2869 & 0.4794 & 0.4493 \\
\hline PR2 & 0.2435 & 0.0237 & 0.0417 & : & $=$ & - & - & - & 1 & $=$ & 0.0851 & - & - \\
\hline & & & & 0.1659 & 0.2162 & 0.1354 & 0.2138 & 0.0585 & & 0.2158 & & 0.1694 & 0.2113 \\
\hline Cl.2 & 0.0892 & 0.1408 & 0.1815 & 0.6918 & 0.6778 & 0.7181 & 0.6863 & 0.6454 & 0.2158 & 1 & 0.206 & 0.6525 & 0.4493 \\
\hline $\mathrm{CN} 2$ & 0.2416 & 0.1345 & 0.1284 & 0.3363 & 0.1778 & 0.0881 & 0.0378 & 0.2869 & 0.0851 & 0.206 & 1 & 0.0896 & 0.1375 \\
\hline IN2 & 0.2285 & 0.2012 & 0.3667 & 0.6268 & 0.6386 & 0.6007 & 0.6082 & 0.4794 & 0.1694 & 0.6525 & 0.0896 & 1 & 0.3675 \\
\hline $\mathrm{CF} 2$ & 0.0536 & 0.1509 & 0.1591 & 0.4526 & 0.4741 & 0.3579 & 0.2524 & 0.4493 & 0.2113 & 0.4493 & 0.1375 & 0.3675 & 1 \\
\hline
\end{tabular}

Correlaciones momento-producto, $r$ de Pearson $(p=0,05)$ 
B. Análisis comparativo en función a la situación laboral (Cuadro $\mathrm{N}$ o 06)

Para el análisis respectivo de esta área, se considera la condición de Contratado y Nombrado y en el cuadro respectivo se puede encontrar los resultados de la media, desviación estándar y el cálculo estadístico diferencial.

Respecto a la variable estrés laboral, no se observa diferencia significativa entre ambos grupos laborales.

Sin embargo, al analizar los resultados de la escala del clima social laboral, sí se observan diferencia significativa, específicamente en la sub escala de Presión (PR) en la medida que los profesionales de condición laboral nombrados obtienen una media mayor que los contratados, y que puede interpretarse como una mayor presión laboral en los profesionales nombrados

C. Análisis comparativo en función al género (Cuadro N 07)

Para el análisis respectivo de esta área, se considera la condición de Masculino y Femenino y en el cuadro respectivo se puede encontrar los resultados de la media, desviación estándar y el cálculo estadístico diferencial.

En ambas variables, referidas al estrés laboral y al clima social laboral, no se observan diferencias estadísticamente significativas, por lo que estas comparaciones no debieran ser tomadas en cuenta como aspecto diferencial en investigaciones futuras.

CUADRO N ${ }^{\circ}$ 06: Comparación de las variables estrés laboral (burnout) y percepción del clima social laboral en función a la situación laboral

\begin{tabular}{lccccc}
\hline \multicolumn{1}{c}{ ÁREAS MEDIDAS } & \multicolumn{2}{c}{ Contratado } & \multicolumn{2}{c}{ Nombrado } & \multirow{2}{*}{ "t" } \\
\cline { 2 - 5 } & X & D.S. & X & D.S. & \\
\hline I. Estrés laboral (burnout) & & & & & \\
1. Cansancio emocional (C.E.) & 14.7 & 11.3 & 14.5 & 9.4 & 0.2641 \\
2. Despersonalización (Dp) & 6.67 & 4.47 & 6.77 & 5.27 & -0.0920 \\
3. Falta de Autorrea1ización personal (Rp) & 8.97 & 7.27 & 6.89 & 6.50 & 1.4126 \\
II. Clima Social Laboral & & & & & \\
\hline A. Relaciones & & & & & \\
1. Implicación (IM) & 4.05 & 2.29 & 4.87 & 2.51 & -1.5594 \\
2. Cohesión (CO) & 3.5 & 1.95 & 3.62 & 1.88 & -0.2926 \\
3. Apoyo (AP) & 2.85 & 2.04 & 3.60 & 2.23 & -1.6005 \\
B. Autorrealización & & & & & \\
4. Autonomía (AV) & 3.41 & 2.18 & 4.31 & 2.24 & -1.8716 \\
5. Organización (OR) & 4.35 & 1.99 & 4.63 & 2.25 & -0.6099 \\
6. Presión (PR) & 3.97 & 1.80 & 4.86 & 1.60 & $\mathbf{- 2 . 4 5 7 4}$ \\
C. Estabilidad y Cambio & & & & & \\
7. Claridad (CL) & 3.88 & 2.44 & 4.22 & 2.68 & -0.6080 \\
8. Control (CN) & 5.08 & 1.92 & 4.58 & 1.91 & 1.2123 \\
9. Innovación (IN) & 3.02 & 2.11 & 3.84 & 2.37 & -1.6545 \\
10.Comodidad (CF) & 4.32 & 2.49 & 3.58 & 2.13 & 1.5009 \\
\hline
\end{tabular}

Nivel de Confianza al 0.05 


\section{CUADRO N ${ }^{\circ}$ 07: Comparación de las variables estrés laboral (burnout) y percepción del clima social laboral en función al sexo}

\begin{tabular}{|c|c|c|c|c|c|}
\hline \multirow{2}{*}{ ÁREAS MEDIDAS } & \multicolumn{2}{|c|}{ Masculino } & \multicolumn{2}{|c|}{ Femenino } & \multirow{2}{*}{ "tt" } \\
\hline & $\mathbf{X}$ & D.S. & $\mathbf{X}$ & D.S. & \\
\hline I. Estrés laboral (burnout) & & & & & \\
\hline 1. Cansancio emocional (CE.) & 15.07 & 11.61 & 14.41 & 9.36 & 0.2842 \\
\hline 2.Despersonalización (Dp) & 8.26 & 6.36 & 6.07 & 4.20 & 1.9406 \\
\hline $\begin{array}{l}\text { Falta de Autorrealización } \\
\text { personal }(\mathrm{Rp})\end{array}$ & 8.34 & 8.01 & 7.37 & 6.30 & 0.6172 \\
\hline II. Clima Social Laboral & & & & & \\
\hline A. Relaciones & 3.88 & 2.48 & 4.89 & 2.41 & -1.7945 \\
\hline 1.Implicación (IM) & 3.15 & 1.99 & 3.79 & 1.87 & -1.4428 \\
\hline 2.Cohesión (CO) & 2.73 & 1.99 & 3.61 & 2.25 & -1.7442 \\
\hline 3.Aроуо (AP) & & & & & \\
\hline B. Autorrealización & & & & & \\
\hline 4.Autonomía (AU) & 3.34 & 2.15 & 4.25 & 2.52 & -1.7652 \\
\hline 5.Organización (OR) & 4.34 & 2.34 & 4.64 & 2.09 & -0.5904 \\
\hline 6.Presión (PR) & 4.34 & 1.64 & 4.61 & 1.74 & -0.6680 \\
\hline C. Estabilidad y Cambio & & & & & \\
\hline 7.Claridad (CL) & 3.46 & 2.31 & 4.40 & 2.68 & -1.5729 \\
\hline 8.Control (CN) & 4.30 & 1.78 & 4.97 & 1.94 & -1.5067 \\
\hline 9. Innovación (IN) & 3.38 & 2.28 & 3.65 & 2.34 & -0.5058 \\
\hline 10.Comodidad (CF) & 3.69 & 2.67 & 3.92 & 2.11 & -0.4409 \\
\hline
\end{tabular}

Nivel de Confianza al 0.05

B. Análisis comparativo en función a actividades profesionales diferenciadas Para la realización del presente análisis comparativo, se tendrá en cuenta los datos obtenidos de los cuadros $\mathrm{N}^{\circ} 08$, de la «A» hasta la «N», cuyos resultados son como a continuación se detallan:

- Entre médicos y enfermeras, no se encuentra diferencia significativa al analizar ambas variables, en la medida que el clima social laboral y el estrés laboral son percibidos en la misma dimensión por ambos profesionales. (Cuadro $\mathrm{N}^{\circ}$ 08-A).

- Entre médicos y obstetrices, no se encuentra diferencia significativa al analizar la variable estrés laboral, pero sí se observan dichas diferencias al analizar la variable clima social laboral, específicamente en el subtest organización (OR), en la que los médicos logran una media mayor en comparación a las obstetrices, lo que se puede interpretar como una mayor preferencia a la organización del trabajo de parte de los médicos. (Cuadro $\mathrm{N}^{\circ}$ 08B)

- Entre médicos y psicólogos, se encuentra diferencia significativa en la variable estrés laboral, específicamente en la subprueba falta de autorrealización personal (RP), en la que los médicos obtienen una media mayor que los psicólogos, es decir, perciben una mayor presión en el ambiente hospitalario. En la variable cima social laboral no se observan diferencias significativas entre ambos grupos de profesionales. (Cuadro $\mathrm{N}^{\circ}$ 08-C).

- Entre médicos y trabajadores sociales se encuentra diferencia significativa a través de la prueba de estrés laboral en sus diferentes subescalas: cansancio emocional (CE), despersonalización (DP) y falta de autorrealización personal (RP); en las cuales, la 
media alcanzada por los médicos es mayor que la alcanzada por los trabajadores sociales. Esto nos indica que la condición de trabajo experimentado por los médicos genera un mayor estrés laboral que los trabajadores sociales. En cuanto a la variable clima social laboral también se observan diferencias estadísticamente significativas, específicamente en el subtest Control $(\mathrm{CN})$, en la que los médicos alcanzan una media mayor a la alcanzada por los trabajadores sociales. (Cuadro $\mathrm{N}^{\circ} 08-\mathrm{D}$ ).

- Entre los médicos y tecnólogos médicos no se encuentra diferencia significativa en la escala estrés laboral, sin embargo, si se observan diferencias en la escala clima social laboral, específicamente en las subescalas implicación (IM), apoyo (AP) y control (CM), con una media mayor en los médicos, por lo que podemos inferir que estos profesionales perciben un mayor compromiso con su trabajo y apoyo de la institución de salud hacia ellos, pero también perciben un mayor control. (Cuadro $\mathrm{N}^{\circ} 08-\mathrm{E}$ )

- Entre las enfermeras y las obstetrices, se encuentra diferencia significativa en la escala de estrés laboral, específicamente en la sub-escala de despersonalización (DP), con una media mayor en las enfermeras. Sin embargo, en la escala clima social laboral no se encuentra diferencia significativa entre ambos grupos. (Cuadro $\mathrm{N}^{\circ} 08-\mathrm{F}$ )

- Entre enfermeras y psicólogos, no se encuentra diferencia significativa en ambas variables de estudio. (Cuadro $\mathrm{N}^{\circ} \mathrm{O} 8-\mathrm{G}$ )

- Entre las enfermeras y los trabajadores sociales se encuentra diferencia significativa en la escala de estrés laboral, específicamente en la escala de despersonalización (DP), donde las enfermeras alcanzan una media mayor en comparación a los trabajadores sociales. Sin embargo en la escala de clima social laboral no se observan diferencias estadísticamente significativas. (Cuadro $\mathrm{N}^{\circ} \mathrm{O} 8-\mathrm{H}$ )

- Entre las enfermeras y tecnólogos médicos, no se encuentra diferencia significativa a través de la escala de estrés laboral, pero si se observan diferencia significativa en la escala de clima social laboral, específicamente en la subescala de implicación (IM) e innovación (IN), con una media mayor en las enfermeras en función a los tecnólogos médicos. (Cuadro $\mathrm{N}^{\circ}$ 08-I)

- Entre las obstetrices y los psicólogos, no se encuentra diferencia significativa a través de ambas variables motivo de estudio. (Cuadro $\mathrm{N}^{\circ} 08-\mathrm{J}$ )

- Entre obstetrices y trabajadores sociales, se encuentra diferencia significativa en la escala de estrés laboral, específicamente en la subescala cansancio emocional (CE) con una media mayor en las obstetrices en comparación a los trabajadores sociales. Sin embargo, en la escala de clima social laboral no se observan diferencias significativas. (Cuadro $\mathrm{N}^{\circ} 08-\mathrm{K}$ )

- Entre obstetrices y tecnólogos médicos, se encuentra diferencia significativa en la escala de estrés laboral, específicamente en la subescala de despersonalización (DP) y falta de autorrealización personal (RP) con una media mayor en los tecnólogos médicos en función a las obstetrices. Sin embargo en la escala de clima social laboral no se observan diferencias estadísticamente significativas. (Cuadro $\mathrm{N}^{\circ} 08-\mathrm{L}$ )

- Entre los psicólogos y trabajadores sociales, no se encuentra diferencia significativa en ambas escalas. (Cuadro $\mathrm{N}^{\circ}$ 08-LL)

- Entre los psicólogos y tecnólogos médicos, se encuentra diferencia significativa, específicamente en la subescala de despersonalización (DP) y falta de autorrealización personal (RP), donde los tecnólogos médicos alcanzan una media mayor en relación a los psicólogos. Igualmente se encuentran diferencia significativa en la escala de clima social laboral, en especial en la subescala de apoyo (AP) e innovación (IN) con una media mayor en este caso, de parte de los psicólogos. (Cuadro $\mathrm{N}^{\circ}$ 08-M) 
- Entre los trabajadores sociales y los tecnólogos médicos, se encuentra diferencia significativa en la escala de estrés laboral en las tres subescalas con una media mayor en los tecnólogos médicos en función a los trabajadores sociales. Igualmente se encuentra diferencia significativa en la escala de clima social laboral, específicamente en la subescala de implicación (1M), con una media mayor en este caso, de los trabajadores sociales en función a los tecnólogos médicos. (Cuadro $\mathrm{N}^{\circ}$ 08-N).

CUADRO N ${ }^{\circ}$ 08-A: Comparación de las variables estrés laboral y percepción del clima social laboral en función a actividades profesionales

Médico - Enfermera

\begin{tabular}{lccccc}
\hline \multirow{2}{*}{\multicolumn{1}{c}{ ÁREAS MEDIDAS }} & \multicolumn{2}{c}{ Médico } & \multicolumn{2}{c}{ Enfermera } & \multirow{2}{*}{ “t” } \\
\cline { 2 - 5 } & $\mathbf{X}$ & D.S. & X & D.S. & \\
\hline I. ESTRÉS LABORAL (burnout) & & & & & \\
1. Cansancio emocional (C.E.) & 16.85 & 9.12 & 13.41 & 9.59 & 1.0172 \\
2. Despersonalización (Dp) & 7.0 & 4.75 & 7.17 & 4.03 & -0.1118 \\
3. Falta de Autorrealización personal (Rp) & 9.0 & 6.81 & 7.05 & 5.27 & 0.8943 \\
\hline II. CLIMA SOCIAL LABORAL & & & & & \\
\hline A. Relaciones & & & & & \\
1. Implicación (IM) & 5.57 & 2.68 & 5.58 & 2.42 & -0.0183 \\
2. Cohesión (CO) & 3.85 & 2.24 & 4.0 & 2.09 & -0.1829 \\
3. Apoyo (AP) & 4.35 & 2.53 & 3.52 & 1.94 & 1.0312 \\
B. Autorrealización & & & & & \\
4. Autonomía (AV) & 3.78 & 2.60 & 4.05 & 2.51 & -0.2961 \\
5. Organización (OR) & 5.64 & 2.09 & 4.88 & 2.44 & 0.9174 \\
6. Presión (PR) & 4.71 & 1.77 & 4.70 & 1.49 & 0.0143 \\
C. Estabilidad y Cambio & & & & & \\
7. Claridad (CL) & 4.71 & 2.89 & 4.52 & 3.06 & 0.1713 \\
8. Control (CN) & 5.71 & 1.36 & 5.17 & 2.18 & 0.8306 \\
9. Innovación (IN) & 3.92 & 2.81 & 4.29 & 2.16 & -0.3656 \\
10.Comodidad (CF) & 4.57 & 2.53 & 3.82 & 2.86 & 1.0191 \\
\hline
\end{tabular}


CUADRO $\mathrm{N}^{\circ}$ 08-B: Comparación de las variables estrés laboral y percepción del clima social laboral en función a actividades profesionales

Médico - Obstetriz

\begin{tabular}{lccccc}
\hline \multirow{2}{*}{\multicolumn{1}{c}{ ÁREAS MEDIDAS }} & \multicolumn{2}{c}{ Médico } & \multicolumn{2}{c}{ Obstetriz } & \multirow{2}{*}{ "t" } \\
\cline { 2 - 5 } & $\mathbf{X}$ & D.S. & X & D.S. & \\
\hline I. Estrés Laboral (burnout) & 16.85 & 9.12 & 12.62 & 7.60 & 1.3859 \\
1. Cansancio emocional (C.E.) & 7.0 & 4.75 & 4.43 & 3.11 & 1.7664 \\
2. Despersonalización (Dp) & 9.0 & 6.81 & 5.81 & 5.81 & 1.3830 \\
3. Falta de Autorrealización personal (Rp) & & & & & \\
\hline II. Clima Social Laboral & & & & & \\
\hline A. Relaciones & 5.57 & 2.68 & 4.25 & 1.77 & 1.6123 \\
1. Implicación (IM) & 3.85 & 2.24 & 3.56 & 1.86 & 0.3927 \\
2. Cohesión (CO) & 4.35 & 2.53 & 2.87 & 2.52 & 1.6019 \\
3. Apoyo (AP) & 3.78 & 2.60 & 3.93 & 2.17 & -0.1738 \\
B. Autorrealización & 5.64 & 2.09 & 3.62 & 1.36 & 3.1652 \\
4. Autonomía (AU) & 4.71 & 1.77 & 4.68 & 1.95 & 0.0390 \\
5. Organización (OR) & & & & & \\
6. Presión (PR) & 4.71 & 2.89 & 3.68 & 2.30 & 1.0819 \\
C. Estabilidad y Cambio & 5.71 & 1.36 & 4.5 & 2.42 & 1.7146 \\
7. Claridad (CL) & 3.92 & 2.81 & 3.31 & 1.95 & 0.7034 \\
8. Control (CN) & 4.57 & 2.53 & 2.93 & 2.01 & 1.9662 \\
9. Innovación (IN) & & & & &
\end{tabular}

CUADRO $\mathrm{N}^{\circ}$ 08-C : Comparación de las variables estrés laboral y percepción del clima social laboral en función a actividades profesionales

Médico - Psicólogo

\begin{tabular}{lccccc}
\hline \multirow{2}{*}{\multicolumn{1}{c}{ ÁREAS MEDIDAS }} & \multicolumn{1}{c}{ Médico } & \multicolumn{2}{c}{ Psicólogo } & \multirow{2}{*}{ "t" } \\
\cline { 2 - 5 } & $\mathbf{X}$ & D.S. & $\mathbf{X}$ & D.S. & \\
\hline I. Estrés Laboral (burnout) & & & & & \\
1. Cansancio emocional (C.E.) & 16.85 & 9.12 & 11.7 & 9.52 & -0.6044 \\
2. Despersonalización (Dp) & 7.0 & 4.75 & 5.1 & 4.17 & -0.852 \\
3. Falta de Autorrealización personal (Rp) & 9.0 & 6.81 & 3.8 & 3.55 & $\mathbf{2 . 0 3 5 5 *}$ \\
\hline II. Clima Social Laboral & & & & & \\
A. Relaciones & & & & & \\
1. Implicación (IM) & 5.57 & 2.68 & 4.3 & 2.35 & -0.5295 \\
2. Cohesión (CO) & 3.85 & 2.24 & 3.1 & 2.02 & 0.2455 \\
3. Apoyo (AP) & 4.35 & 2.53 & 4.0 & 2.0 & -0.2350 \\
B. Autorrealización & & & & & \\
4. Autonomía (AU) & 3.78 & 2.60 & 4.8 & 2.14 & -0.2415 \\
5. Organización (OR) & 5.64 & 2.09 & 4.3 & 1.88 & 0.5274 \\
6. Presión (PR) & 4.71 & 1.77 & 4.9 & 0.99 & 1.0326 \\
C. Estabilidad y Cambio & & & & & \\
7. Claridad (CL) & 4.71 & 2.89 & 3.8 & 2.65 & -0.9286 \\
8. Control (CN) & 5.71 & 1.36 & 4.4 & 1.71 & 0.5291 \\
9. Innovación (IN) & 3.92 & 2.81 & 4.3 & 2.45 & 0.7045 \\
10.Comodidad (CF) & 4.57 & 2.53 & 3.3 & 2.21 & 1.2657 \\
\hline *Significativo al 0.05 & & & & &
\end{tabular}


CUADRO N ${ }^{\circ}$ 08-D: Comparación de las variables estrés laboral y percepción del clima social laboral en función a actividades profesionales

Médico - Trabajador Social

\begin{tabular}{|c|c|c|c|c|c|}
\hline \multirow{2}{*}{ ÁREAS MEDIDAS } & \multicolumn{2}{|c|}{ Médico } & \multicolumn{2}{|c|}{ Trab. Social } & \multirow{2}{*}{$" t "$} \\
\hline & $\mathbf{X}$ & D.S. & $\mathbf{X}$ & D.S. & \\
\hline \multicolumn{6}{|l|}{ I. Estrés Laboral (burnout) } \\
\hline 1. Cansancio emocional (C.E.) & 16.85 & 9.12 & 6.12 & 5.33 & $3.0261 *$ \\
\hline 2. Despersonalización (Dp) & 7.0 & 4.75 & 2.5 & 2.97 & $2.4065 *$ \\
\hline 3. Falta de Autorrealizaci6n personal (Rp) & 9.0 & 6.81 & 2.75 & 4.13 & $2.3446^{*}$ \\
\hline \multicolumn{6}{|l|}{ II. CLIMA SOCIAL LABORAL } \\
\hline \multicolumn{6}{|l|}{ A. Relaciones } \\
\hline 1. Implicación (IM) & 5.57 & 2.68 & 5.87 & 2.79 & -0.2515 \\
\hline 2. Cohesión (CO) & 3.85 & 2.24 & 4.12 & 2.16 & -0.2722 \\
\hline 3. Apoyo (AP) & 4.35 & 2.53 & 4.25 & 2.76 & 0.0924 \\
\hline \multicolumn{6}{|l|}{ B. Autorrealización } \\
\hline 4. Autonomía (AU) & 3.78 & 2.60 & 4.12 & 2.03 & -0.3161 \\
\hline 5. Organización (OR) & 5.64 & 2.09 & 4.75 & 2.31 & 0.9257 \\
\hline 6. Presi6n (PR) & 4.71 & 1.77 & 4.5 & 2.13 & 0.2533 \\
\hline \multicolumn{6}{|l|}{ C. Estabilidad y Cambio } \\
\hline 7. Claridad (CL) & 4.71 & 2.89 & 4.37 & 2.55 & 0.2752 \\
\hline 8. Control (CN) & 5.71 & 1.36 & 4.12 & 1.55 & $2.7611^{*}$ \\
\hline 9. Innovaci6n (IN) & 3.92 & 2.81 & 3.87 & 2.16 & 0.0463 \\
\hline 10.Comodidad (CF) & 4.57 & 2.53 & 4.75 & 2.86 & -0.1517 \\
\hline
\end{tabular}

* Significativo al 0.05

CUADRO N $^{\circ}$ 08-E: Comparación de las variables estrés laboral y percepción del clima social laboral en función a actividades profesionales

Médico - Tecnólogo Médico

\begin{tabular}{|c|c|c|c|c|c|}
\hline \multirow{2}{*}{ ÁREAS MEDIDAS } & \multicolumn{2}{|c|}{ Médico } & \multicolumn{2}{|c|}{ Tec. Médico } & \multirow{2}{*}{ "t" } \\
\hline & $\mathbf{X}$ & D.S. & $\mathbf{X}$ & D.S. & \\
\hline \multicolumn{6}{|l|}{ I. Estrés Laboral (burnout) } \\
\hline 1. Cansancio emocional (C. E.) & 16.85 & 9.12 & 18.78 & 11.26 & -0.5550 \\
\hline 2. Despersonalización (Dp) & 7.0 & 4.75 & 9.28 & 5.80 & -1.2729 \\
\hline 3. Falta de Autorrealización personal (Rp) & 9.0 & 6.81 & 11.14 & 7.92 & -0.8630 \\
\hline \multicolumn{6}{|l|}{ II. Clima Social Laboral } \\
\hline $\begin{array}{l}\text { A. Relaciones } \\
\text { 1. Implicación (IM) }\end{array}$ & 5.57 & 2.68 & 3.5 & 2.28 & $2.6140 *$ \\
\hline $\begin{array}{l}\text { 2. Cohesión (CO) } \\
\text { 3. Apoyo (AP) }\end{array}$ & $\begin{array}{l}3.85 \\
4.35\end{array}$ & $\begin{array}{l}2.24 \\
2.53\end{array}$ & $\begin{array}{l}3.32 \\
2.57\end{array}$ & $\begin{array}{l}1.63 \\
1.66\end{array}$ & $\begin{array}{l}0.8817 \\
2.7443^{*}\end{array}$ \\
\hline \multicolumn{6}{|l|}{ B. Autorrealización } \\
\hline 4. Autonomía (AU) & 3.78 & 2.60 & 3.78 & 2.16 & $5.8512 *$ \\
\hline 5. Organización (OR) & 5.64 & 2.09 & 4.39 & 2.31 & 1.6998 \\
\hline 6. Presión (PR) & & & & & 09673 \\
\hline $\begin{array}{l}\text { C. Estabilidad y Cambio } \\
\text { 7. Claridad (CL) }\end{array}$ & 4.71 & 2.89 & $\begin{array}{l}4.14 \\
3.92\end{array}$ & $\begin{array}{l}1.81 \\
2.47\end{array}$ & 0.9159 \\
\hline 8. Control (CN) & 5.71 & 1.36 & 4.57 & 1.83 & $2.1276^{*}$ \\
\hline 9. Innovación (IN) & 3.92 & 2.81 & 2.78 & 1.85 & 1.5787 \\
\hline 10.Comodidad (CF) & 4.57 & 2.53 & 4.0 & 2.46 & 0.7019 \\
\hline
\end{tabular}

* Significativo al 0.05 
CUADRO $\mathrm{N}^{\circ}$ 08-F: Comparación de las variables estrés laboral y percepción del clima social laboral en función a actividades profesionales

Enfermera - Obstetriz

\begin{tabular}{|c|c|c|c|c|c|}
\hline \multirow{2}{*}{ ÁREAS MEDIDAS } & \multicolumn{2}{|c|}{ Enfermera } & \multicolumn{2}{|c|}{ Obstetriz } & \multirow{2}{*}{ "t" } \\
\hline & $\mathbf{X}$ & D.S. & $\mathbf{X}$ & D.S. & \\
\hline \multicolumn{6}{|l|}{ I. Estrés Laboral (burnout) } \\
\hline 1. Cansancio emocional (C.E.) & 13.41 & 9.59 & 12.62 & 7.60 & 0.2599 \\
\hline 2. Despersonalización (Dp) & 7.17 & 4.03 & 4.43 & 3.11 & $2.1717 *$ \\
\hline 3. Falta de Autorrealizaci6n personal (Rp) & 7.05 & 5.27 & 5.81 & 5.81 & 0.6458 \\
\hline \multicolumn{6}{|l|}{ II. CLIMA SOCIAL LABORAL } \\
\hline \multicolumn{6}{|l|}{ A. Relaciones } \\
\hline 1. Implicación (IM) & 5.58 & 2.42 & 4.25 & 1.77 & 1.8007 \\
\hline 2. Cohesión (CO) & 4.00 & 2.09 & 3.56 & 1.86 & 0.6333 \\
\hline 3. Apoyo (AP) & 3.52 & 1.94 & 2.87 & 2.52 & 0.8375 \\
\hline \multicolumn{6}{|l|}{ B. Autorrealización } \\
\hline 4. Autonomía (AU) & 4.05 & 2.51 & 3.93 & 2.17 & 0.147 \\
\hline 5. Organización (OR) & 4.88 & 2.44 & 3.62 & 1.36 & 1.8084 \\
\hline 6. Presi6n (PR) & 4.70 & 1.40 & 4.68 & 1.95 & 0.0304 \\
\hline \multicolumn{6}{|l|}{ C. Estabilidad y Cambio } \\
\hline 7. Claridad (CL) & 4.52 & 3.06 & 3.93 & 2.17 & 0.8880 \\
\hline 8. Control $(\mathrm{CN})$ & 5.17 & 2.18 & 3.62 & 1.36 & 0.8431 \\
\hline 9. Innovación (IN) & 4.29 & 2.73 & 4.68 & 1.95 & 1.1795 \\
\hline 10.Comodidad (CF) & 3.82 & 1.50 & 3.93 & 2.17 & 1.4350 \\
\hline
\end{tabular}

* Significativo al 0.05

CUADRO N ${ }^{\circ}$ 08-G: Comparación de las variables estrés laboral y percepción del clima social laboral en función a actividades profesionales

Enfermera - Psicólogo

\begin{tabular}{|c|c|c|c|c|c|}
\hline \multirow{2}{*}{ ÁREAS MEDIDAS } & \multicolumn{2}{|c|}{ Enfermera } & \multicolumn{2}{|c|}{ Psicólogo } & \multirow{2}{*}{$" t "$} \\
\hline & $\mathbf{X}$ & D.S. & $\mathbf{X}$ & D.S. & \\
\hline \multicolumn{6}{|l|}{ I. Estrés Laboral (burnout) } \\
\hline 1. Cansancio emocional (C. E.) & 13.41 & 9.59 & 11.7 & 9.52 & 0.4489 \\
\hline 2. Despersonalización (Dp) & 7.17 & 4.03 & 5.1 & 4.17 & 1.2751 \\
\hline 3. Falta de Autorrealización personal (Rp) & 7.05 & 5.27 & 3.8 & 3.55 & 1.7299 \\
\hline \multicolumn{6}{|l|}{ II. Clima Social Laboral } \\
\hline $\begin{array}{l}\text { A. Relaciones } \\
\text { 1. Implicación (IM) }\end{array}$ & 5.58 & 2.42 & & & \\
\hline 2. Cohesión $(\mathrm{CO})$ & & & & & \\
\hline 3. Apoyo (AP) & & & & & \\
\hline B. Autorrealización & & & & & \\
\hline 4. Autonomía (AU) & & & & & \\
\hline 5. Organización (OR) & & & & & \\
\hline 6. Presión (PR) & & & & & \\
\hline C. Estabilidad y Cambio & & & & & \\
\hline 7. Claridad (CL) & & & & & \\
\hline 8. Control $(\mathrm{CN})$ & & & & & \\
\hline 9. Innovación (IN) & & & & & \\
\hline 10.Comodidad (CF) & & & & & \\
\hline
\end{tabular}


CUADRO $\mathrm{N}^{\circ} 08-\mathrm{H}$ : Comparación de las variables estrés laboral y percepción del clima social laboral en función a actividades profesionales

Enfermera - Trabajador Social

\begin{tabular}{|c|c|c|c|c|c|}
\hline \multirow{2}{*}{ ÁREAS MEDIDAS } & \multicolumn{2}{|c|}{ Enfermera } & \multicolumn{2}{|c|}{ Trabajador Social } & \multirow{2}{*}{ "t" } \\
\hline & $\mathrm{x}$ & D.S. & $\mathrm{x}$ & D.S. & \\
\hline \multicolumn{6}{|l|}{ L. Estrés laboral (burnout) } \\
\hline 1. Cansancio cmocional (C.E.) & 13.41 & 959 & 6.12 & 5,33 & 1.9939 \\
\hline 2. Despersonalización (Dp) & 7.17 & 4.03 & 2.5 & 2.97 & $2.9129^{\circ}$ \\
\hline 3. Falta de Autorrealización personal ( $R \mathrm{p})$ & 705 & 5.27 & 2.75 & 4.13 & 2.0286 \\
\hline \multicolumn{6}{|l|}{ II. Clima Social Laboral } \\
\hline \multicolumn{6}{|l|}{ A. Relaciones } \\
\hline 1. Implicación (IM) & 5.58 & 2.42 & 5.87 & 2.79 & $-0,2627$ \\
\hline 2. Cohesión (CO) & 400 & 2.09 & 4.12 & 2.16 & -0.1378 \\
\hline 3. Apoyo (AP) & 352 & 1.94 & 425 & 2.76 & -0.7557 \\
\hline \multicolumn{6}{|l|}{ B. Autorrealización } \\
\hline 4. Autonomía (AU) & 4.05 & 251 & 4.12 & 2.03 & 0.0649 \\
\hline 5. Organizución (OR) & 4.88 & 2.44 & 4.75 & 2.31 & 0.1282 \\
\hline 6. Presión (PR) & 4.70 & 140 & 4.5 & 2.13 & 0.2802 \\
\hline \multicolumn{6}{|l|}{ C. Estabilidad y Cambio } \\
\hline 7. Claridad (CL) & 4.52 & 3.06 & 4.37 & 2.55 & 0.1233 \\
\hline 8. Control (CN) & 5.17 & 2.18 & 4.12 & 1.55 & 1.2173 \\
\hline 9. Innovación (IN) & 4.29 & 2.73 & 3.87 & 2.16 & 0.3797 \\
\hline 10. Comodidad (CF) & 3.82 & 150 & 4.75 & 2.86 & -1.0690 \\
\hline
\end{tabular}

* Significativo al 0,05

CUADRO N 08-1: Comparación de las variables estrés laboral y percepción del clima social laboral en función a actividades profesionales Enfermera - Tecnólogo Medico

\begin{tabular}{|c|c|c|c|c|c|}
\hline \multirow{2}{*}{ ÁREAS MEDIDAS } & \multicolumn{2}{|c|}{ Enfermera } & \multicolumn{2}{|c|}{ Tec. Medico } & \multirow{2}{*}{$" t "$} \\
\hline & $\mathbf{x}$ & D.S. & $\mathbf{x}$ & D.S. & \\
\hline \multicolumn{6}{|l|}{ I. Estrés laboral (burnout) } \\
\hline 1. Cansancio emocional (CE) & 13.41 & 9.59 & 1878 & 11.26 & -1.6378 \\
\hline 2. Despersonalización $\left(\mathrm{Dp}_{\mathrm{p}}\right)$ & 7.17 & 4.03 & 9.28 & 5,80 & 1.3150 \\
\hline 3. Falta de Autorrealización personal (Rp) & 7.05 & 5.27 & 11.14 & 7.92 & -1.8818 \\
\hline \multicolumn{6}{|l|}{ II. Clima Social Laboral } \\
\hline \multicolumn{6}{|l|}{ A. Relaciones } \\
\hline 1. Implicación (IM) & 5.58 & 2.42 & 3.5 & 2.28 & $2.9044^{*}$ \\
\hline 2. Cohesión $(\mathrm{CO})$ & 4.00 & 209 & 3.32 & 1.63 & 1.2140 \\
\hline 3. Apoyo (AP) & 3.52 & 194 & 2.57 & 1.66 & 1.7578 \\
\hline \multicolumn{6}{|l|}{ B. Autorrealización } \\
\hline 4. Autonomía (AU) & 4.05 & 251 & 3.78 & 2.16 & 0.3860 \\
\hline 5. Organización (OR) & 4.88 & 2.44 & 4.39 & 2.31 & 0.6732 \\
\hline 6. Presión (PR) & 470 & 1.40 & 4.14 & 1.81 & 1.0741 \\
\hline C. Estabilidad y Cambio & & & & $+\quad-2$ & \\
\hline 7. Claridad (CL) & 4.52 & 3.06 & 3.92 & 2.47 & 0.7207 \\
\hline 8 Control (CN) & 5.17 & 2.18 & 4.57 & 1.83 & 0.9975 \\
\hline 9. Innovación (IN) & 4.29 & 2.73 & 2.78 & 1.85 & $2.2081^{*}$ \\
\hline 10.Comodidad (CF) & 3.82 & 150 & 4.0 & 2.46 & -0.2658 \\
\hline
\end{tabular}

+ Significativo al 0.05 
CUADRO No 08-1: Comparación de las variables estrés laboral y percepción del clima social laboral en función a actividades profesionales

Obstretiz-Psicólogo

\begin{tabular}{|c|c|c|c|c|c|}
\hline \multirow{2}{*}{ AREAS MEDIDAS } & \multicolumn{2}{|c|}{ Ohstetriz } & \multicolumn{2}{|c|}{ Psicólogo } & \multirow{2}{*}{ "t" } \\
\hline & $\mathrm{X}$ & D.S. & $\mathrm{X}$ & D.S. & \\
\hline \multicolumn{6}{|l|}{ I. Estrés laboral (burnout) } \\
\hline 1. Cansancio emocional (CE.) & 12.62 & 7.60 & 11.7 & 9.52 & 0.2739 \\
\hline 2. Despersonalización (Dp) & 4.43 & 3.11 & 5.1 & 4.17 & -0.4626 \\
\hline 3. Falta de Autorrealización personal $(\mathrm{Rp})$ & 5.81 & 5.81 & 3.8 & 355 & 0.9822 \\
\hline \multicolumn{6}{|l|}{ II. Clima Social Laboral } \\
\hline \multicolumn{6}{|l|}{ A. Relaciones } \\
\hline 1. Implicación (IM) & 4.25 & 177 & 4.3 & 2.35 & 0.0616 \\
\hline 2. Cohesión $(\mathrm{CO})$ & 3.56 & 1.86 & 3.1 & 2.02 & 0.5963 \\
\hline 3. Apoyo (AP) & 287 & 2.52 & 4.0 & 20 & -1.1911 \\
\hline \multicolumn{6}{|l|}{$\frac{\text { 3. Apoyo (AP) }}{\text { B. Autorrealización }}$} \\
\hline 4. Autonomía (AU) & 3.93 & 2.17 & 4.8 & 2.14 & -0.9880 \\
\hline 5. Organización (OR) & 3.62 & 1.36 & 4.3 & 188 & -1.0603 \\
\hline 6. Presión (PR) & 4.68 & 1.95 & 4.9 & 0.99 & $-0,3170$ \\
\hline \multicolumn{6}{|l|}{ C. Estabilidad y Cambio } \\
\hline 7. Claridad (Cl) & 3.93 & 2.17 & 3.8 & 2.65 & -0.1143 \\
\hline 8. Control (CN) & 362 & 1.36 & 4.4 & 1.71 & 0.1136 \\
\hline 9. Innovación (IN) & 4.68 & 1.95 & 43 & 2.45 & -1.1363 \\
\hline 10.Comodidad (CF) & 3.93 & 2.17 & 3.3 & 2.21 & -0.4296 \\
\hline
\end{tabular}

CUADRO Nº8-K: Comparación de las variables estrés laboral y percepción del clima social laboral en función a actividades profesionales

Obstetriz-Trabajador Social

\begin{tabular}{|c|c|c|c|c|c|}
\hline \multirow{2}{*}{ ÁREAS MEDIDAS } & \multicolumn{2}{|c|}{ Obstetriz. } & \multicolumn{2}{|c|}{ Trabajador Social } & \multirow{2}{*}{$" \mathrm{t} "$} \\
\hline & $\mathbf{X}$ & D.S. & $\mathbf{x}$ & D.S. & \\
\hline \multicolumn{6}{|l|}{ I. Estrés laboral (burnout) } \\
\hline 1. Cansancio emocional (C.E.) & 12.62 & 7.60 & 6.12 & 5.33 & $2.1558^{\circ}$ \\
\hline 2. Despersonalización (Dp) & 4.43 & 3.11 & 25 & 2.97 & 1.4554 \\
\hline 3. Falta de Autonealización persontal (Rp) & 5.81 & 5.81 & 2.75 & 4.13 & 1.3259 \\
\hline \multicolumn{6}{|l|}{ II. Clima Social Laboral } \\
\hline \multicolumn{6}{|l|}{ A. Clima Sockal Laborial } \\
\hline 1. Implicación (IM) & 4.25 & 1.77 & 5.87 & 279 & -1.7439 \\
\hline 2. Cohesión (CO) & 3.56 & 1.86 & 4.12 & 2.16 & -0.6616 \\
\hline 3. Apoyo (AP) & 2.87 & 2.52 & 4.25 & 2.76 & -1.2191 \\
\hline \multicolumn{6}{|l|}{ B. Apoyo (AP) } \\
\hline 4. Autonomia (AU) & 3.93 & 2.17 & 4.12 & 2.03 & -0.2032 \\
\hline 5. Otganización (OR) & 3.62 & 1.36 & 4.75 & 2.31 & -1.5085 \\
\hline 6. Presión (PR) & 4.68 & 1.95 & 4.5 & 2.13 & 0.2147 \\
\hline \multicolumn{6}{|l|}{ C. Estabilidad y Cambio } \\
\hline 7. Claridad (Cl) & 3.93 & 2.17 & 4.37 & 2.55 & -0.6652 \\
\hline 8. Control (CN) & 3.62 & 1.36 & 4.12 & 1.55 & 0,3966 \\
\hline 9. Innovación (IN) & 4.68 & 1.95 & 3.87 & 2.16 & -05411 \\
\hline 10.Comodidad (CF) & 3,93 & 2.17 & 4.75 & 2.86 & -18040 \\
\hline
\end{tabular}

- Significativo al 0.05 
CUADRO N 08-L: Comparación de las variables estrés laboral y percepción del clima social laboral en función a actividades profesionales

Obstetriz-Tecnólogo Médico

\begin{tabular}{|c|c|c|c|c|c|}
\hline \multirow{2}{*}{ ÁREAS MEDIDAS } & \multicolumn{2}{|c|}{ Obstetriz } & \multicolumn{2}{|c|}{ Tec. Médico } & \multirow{2}{*}{ "t" } \\
\hline & $\mathrm{X}$ & D.S. & $\mathbf{x}$ & D.S. & \\
\hline \multicolumn{6}{|l|}{ 1. Fstrés laboral (burnout) } \\
\hline 1. Cansancio emocional (C.E.) & 12.62 & 7.60 & 18.78 & 11.26 & -1.9445 \\
\hline 2. Despersonalizaciōn (Dp) & 4.43 & 3.11 & 9,28 & 5.80 & -3.0857 \\
\hline 3. Falta de Autorrealización personal (Rp) & 5.81 & 5.81 & 11.14 & 7.92 & -2.3480 \\
\hline \multicolumn{6}{|l|}{ II. Clima Social Laboral } \\
\hline \multicolumn{6}{|l|}{ A. Relaciones } \\
\hline 1. Implicación (IM) & 4.25 & 1.77 & 3.5 & 2.28 & 1.1311 \\
\hline 2. Cohesían (CO) & 356 & 1.86 & 3.32 & 1.63 & 0.4476 \\
\hline 3. Apoyo (AP) & 2.87 & 252 & 257 & 1.66 & 0.4806 \\
\hline \multicolumn{6}{|l|}{ B. Autorrealización } \\
\hline 4. Autonomia (AU) & 393 & 2.17 & 3.78 & 2.16 & 0.2232 \\
\hline 5. Organización (OR) & 3.62 & 1.36 & 4.39 & 231 & -1.2092 \\
\hline 6. Presión (PR) & 4.68 & 1.95 & 4.14 & 1.81 & 0.9293 \\
\hline \multicolumn{6}{|l|}{ C. Estabilidad y Cambio } \\
\hline 7. Claridad (CL) & 3.93 & 2.17 & 3.92 & 2.47 & -0.3183 \\
\hline 8. Control $(\mathrm{CN})$ & 3.62 & 1.36 & 4.57 & 1.83 & -0.1104 \\
\hline 9. Innovación (IN) & 4.68 & 1.95 & 2.78 & 1.85 & 0.8890 \\
\hline 10. Comodidad (CF) & 3.93 & 2.17 & 4.0 & 246 & -1.4649 \\
\hline
\end{tabular}

- Significative al 0.05

CUADRO N08-L.L: Comparación de las variables estrés laboral y percepción del clima social laboral en función a actividades profesionales Psicólogo-Trabajador Social

\begin{tabular}{|c|c|c|c|c|c|}
\hline \multirow{2}{*}{ AREAS MEDIDAS } & \multicolumn{2}{|c|}{ Psicólogo } & \multicolumn{2}{|c|}{ Trabajador Social } & \multirow{2}{*}{$" t "$} \\
\hline & $\mathbf{X}$ & D.S. & $\mathbf{x}$ & D.S. & \\
\hline \multicolumn{6}{|l|}{ L. Estrés laboral (burnout) } \\
\hline 1. Cansancio emocional (C.E.) & 11.7 & 9.52 & 6.12 & 5.33 & 1.4756 \\
\hline 2. Despersonalización (Dp) & 5.1 & 4.17 & 2.5 & 2.97 & 1.4819 \\
\hline 3. Falta de Autorrealización personal (Rp) & 3.8 & 3.55 & 2.75 & 4.13 & 0.5788 \\
\hline \multicolumn{6}{|l|}{ II. Clima Social Laboral } \\
\hline \multicolumn{6}{|l|}{ A. Relaciones } \\
\hline 1. Implicación (IM) & 4.3 & 2.35 & 5.87 & 2.79 & -1.2963 \\
\hline 2. Cohesión (CO) & 3.1 & 2.02 & 4.12 & 2.16 & -1.0347 \\
\hline 3. Apoyo (AP) & 4.0 & 2.0 & 4.25 & 2.76 & -0.2228 \\
\hline \multicolumn{6}{|l|}{ B. Autorrealización } \\
\hline 4. Autonomía (AU) & 4.8 & 2.14 & 4.12 & 2.03 & 0.6780 \\
\hline 5. Organización (OR) & 43 & 1.88 & 4.75 & 2.31 & -0.4548 \\
\hline 6. Presión (PR) & 49 & 0.99 & 4.5 & 2.13 & 0.5274 \\
\hline \multicolumn{6}{|l|}{ C. Estabilidad y Cambio } \\
\hline 7. Claridad (CL) & 3.8 & 2.65 & 4.37 & 2.55 & -0.4634 \\
\hline 8. Control (CN) & 44 & 1.71 & 4.12 & 155 & 0.3525 \\
\hline 9. Innovación (IN) & 43 & 245 & 3.87 & 2.16 & 0.3842 \\
\hline 10. Comodidad (CF) & 3.3 & 2.21 & $\begin{array}{r}4.75 \\
\end{array}$ & 2.86 & -12130 \\
\hline
\end{tabular}


CUADRO No 08-M: Comparación de las variables estrés laboral y percepción del clima social laboral en función a actividades profesionales

Psicólogo-Tecnólogo Médico

\begin{tabular}{|c|c|c|c|c|c|}
\hline \multirow{2}{*}{ ÁREAS MEDIDAS } & \multicolumn{2}{|c|}{ Psicólogo } & \multicolumn{2}{|c|}{ Tee. Médico } & \multirow{2}{*}{ "t" } \\
\hline & $\mathbf{x}$ & D.S. & $\mathbf{x}$ & D.S. & \\
\hline \multicolumn{6}{|l|}{ L. Estrés laboral (burnout) } \\
\hline 1. Cansancio emocional (C.E.) & 11.7 & 9.52 & 18.78 & 11.26 & -1.7721 \\
\hline 2. Despersonalización (Dp) & 5.1 & 4.17 & 9.28 & 580 & $-2.0873^{\circ}$ \\
\hline 3. Falta de Autorrealización personal (Rp) & 3.8 & 3.55 & 11.14 & 7.92 & $-2.8102^{6}$ \\
\hline \multicolumn{6}{|l|}{ I. Clima Social Laboral } \\
\hline \multicolumn{6}{|l|}{$\begin{array}{l}\text { I. Clima Social Laboral } \\
\text { A. Relaciones }\end{array}$} \\
\hline 1. Implicación (IM) & 4.3 & 2.35 & 3.5 & 2.28 & 0.9425 \\
\hline 2. Cohesion (CO) & 3.1 & 2.02 & 3.32 & 1.63 & -0.3454 \\
\hline 3. Apoyo (AP) & 40 & 2.0 & 2.57 & 1.66 & $2.2098^{\circ}$ \\
\hline \multicolumn{6}{|l|}{ B. Apoyo (AP) } \\
\hline 4. Autonomfa (AU) & 48 & 2.14 & 3.78 & 2.16 & 1.2733 \\
\hline 5 Organización (OR) & 4.3 & 1.88 & 4.39 & 2.31 & -0.1137 \\
\hline 6. Presión (PR) & 49 & 0.99 & 4.14 & 1.81 & 1.2435 \\
\hline \multicolumn{6}{|l|}{ C. Estabilidad y Cambio } \\
\hline 7. Claridad (CL) & 3.8 & 2.65 & 392 & 247 & -0.1382 \\
\hline 8. Control (CN) & 4.4 & 1.71 & 4.57 & 1.83 & -0.2578 \\
\hline 9. Innovación (IN) & 4.3 & 2.45 & 2.78 & 1.85 & $2.0354^{*}$ \\
\hline 10. Comodidad (CF) & 33 & 2.21 & 4.0 & 2.46 & -0.7903 \\
\hline
\end{tabular}

- Significativo al 0.05

CUADRO N $N^{\circ} 08-\mathrm{N}$ : Comparación de las variables estrés laboral y percepción del clima social laboral en función a actividades profesionales

Trabajador Social - Tecnólogo Médico

\begin{tabular}{|c|c|c|c|c|c|}
\hline \multirow{2}{*}{ ÁREAS MEDHDAS } & \multicolumn{2}{|c|}{ Trabajo Social } & \multicolumn{2}{|c|}{ Tec, Médico } & \multirow{2}{*}{$" t "$} \\
\hline & $\mathbf{x}$ & D.S. & $\mathbf{x}$ & D.S. & \\
\hline \multicolumn{6}{|l|}{ I. Fstrés laboral (burnout) } \\
\hline 1. Cansancio emocional (C.E.) & 6.12 & 533 & 18.78 & 11.26 & $-3.0591^{\prime \prime}$ \\
\hline Despersonalización $\left(D_{p}\right)$ & 2.5 & 2.97 & 9.28 & 5.80 & $-3.1662^{7}$ \\
\hline 3. Falta de Autorrealización personal (Rp) & 2.75 & 4.13 & 11.14 & 7.92 & $-2.8638^{\circ}$ \\
\hline \multicolumn{6}{|l|}{ I. Clima Social Laboral } \\
\hline \multicolumn{6}{|l|}{ 1. Relaciones } \\
\hline 1. Implicación (IM) & 5.87 & 2.79 & 35 & 2.28 & $2.4682^{\circ}$ \\
\hline 2. Cobesión $(\mathrm{CO})$ & 4.12 & 2.16 & 3,32 & 1.63 & 1.1407 \\
\hline 3. Apoyo (AP) & 4.25 & 276 & 2.57 & 1.66 & 2.1549 \\
\hline \multicolumn{6}{|l|}{$\begin{array}{l}\text { 3. Apoyo (AP) } \\
\text { B. Autorrealización }\end{array}$} \\
\hline 4. Autonornia $(\mathrm{AU})$ & 4.12 & 2.03 & 3.78 & 2.16 & 0.3956 \\
\hline 5. Organización (OR) & 4.75 & 2.31 & 4.39 & 231 & 0.3848 \\
\hline 6. Presión (PR) & 45 & 2.13 & 4.14 & 1.81 & 0.4714 \\
\hline \multicolumn{6}{|l|}{ C. Estabilidad y Cambio } \\
\hline 7. Clandad (CL) & 437 & 2.55 & 3.92 & 2.47 & 0.4462 \\
\hline 8. Control (CN) & 4.12 & 1.55 & 4.57 & 1.83 & -06255 \\
\hline 9. Innovación (IN) & 3.87 & 2.16 & 278 & 1.85 & 1,4137 \\
\hline 10. Comodidad (CF) & 4.75 & 2.86 & 4.0 & 2.46 & 0.7329 \\
\hline
\end{tabular}

* Significative al 0.05 


\section{B. Análisis comparativo en función al tiempo de servicio}

Para la realización del presente análisis comparativo, se tuvo en cuenta los datos presentados en el cuadro $\mathrm{N}$ o 02 , en la cual se puede observar la distribución de la muestra en cuatro grupos:

- Grupo A: Tiempo de servicio entre 1 y 8 años.

- Grupo B: Tiempo de servicio entre 9 y 16 años.

- Grupo C: Tiempo de servicio entre 17 y 24 años.

- Grupo D: Tiempo de servicio entre 25 y 32 años.

Al realizar el análisis comparativo entre los grupos mencionados, obtenemos lo siguiente:

- Grupo A - Grupo B: En la escala de estrés laboral, no se observan diferencias estadísticamente significativas, sin embargo en la escala de clima social laboral sí se observan dichas diferencias, especialmente en la subescala Presión (PR), donde el grupo B alcanza una media mayor al del grupo A. Estos resultados pueden ser interpretados como un indicador de que a mayor tiempo de servicio se percibe una mayor presión de parte de la institución hacia los trabajadores. (Cuadro No 09-A).

- Grupo A - Grupo C: No se observan diferencias significativas entre ambos grupos, tanto en la escala de estrés laboral como en la escala de clima social laboral. (Cuadro No 09B)

- Grupo A-Grupo D: Sí se observan diferencias estadísticamente significativas en la escala de estrés laboral, específicamente en la subescala.

Falta de Autorrealización Personal (RP), con una media mayor para el grupo D en comparación al grupo A. Podríamos pensar a partir de estos resultados que los profesionales de la salud, a mayor tiempo de servicio desarrollan un mayor estrés laboral, con manifestaciones de desmotivación. Sin embargo, en la escala de clima social laboral no se observan diferencias significativas. (Cuadro $\mathrm{N}^{\circ} 09 \mathrm{C}$ )

- Grupo B - Grupo $C$ : No se observan diferencias significativas en ambos grupos, tanto en la escala de estrés laboral como en la esc.ala de clima social laboral. (Cuadro $\mathrm{N}^{\circ}$ 09-D)

- Grupo B - Grupo D: Se observan diferencias estadísticamente significativas en la escala de estrés laboral en ambos grupos, específicamente en la subescala de Falta de Realización Personal (RP), con una media mayor en el grupo D, reiterando nuevamente que a mayor tiempo de servicio, mayor agotamiento personal. En la escala de clima social laboral observamos diferencias significativas en la subescala de Presión (PR), con una media mayor, en este caso, del grupo B. Habría que pensar que el grupo D no se siente presionado por la institución, pero sí se siente agotado por las actividades laborales real izadas. (Cuadro $\mathrm{N}^{\circ}$ 09-E)

- Grupo C - Grupo D: Se observan diferencias significativas en la escala de estrés laboral, específicamente en la subescala de Falta de realización personal (RP) con una media mayor en el grupo D. En la escala de Clima social laboral no se observan diferencias significativas. (Cuadro $\mathrm{N}^{\circ}$ 09-F) 
CUADRO N09-A: Comparación de las variables estrés laboral y percepción del clima social laboral en función tiempo de servicio

$$
\text { Grupo } * \mathrm{~A} *-\mathrm{Grupo} * \mathrm{~B} \text { : }
$$

\begin{tabular}{|c|c|c|c|c|c|}
\hline \multirow{2}{*}{ AREAS MEDIDAS } & \multicolumn{2}{|c|}{ Grupo " $\mathbf{A}$ " } & \multicolumn{2}{|c|}{ Grupo "B" } & \multirow{2}{*}{$" t "$} \\
\hline & $\mathrm{x}$ & D.S. & $\mathrm{x}$ & D.S. & \\
\hline \multicolumn{6}{|l|}{ L. Fstrés baboral (burnout) } \\
\hline 1. Cansancio emocional (C.E.) & 13.37 & 8.64 & 16.96 & 10.13 & -1.4811 \\
\hline 2. Despersonalización (Dp) & 6.53 & 4.89 & 757 & 4.78 & -0.8298 \\
\hline 3. Falta de Autorrealización personal $(R p)$ & 7.5 & 7.32 & 6.92 & 8.25 & 0.3224 \\
\hline \multicolumn{6}{|l|}{ IL. Clima Social Laboral } \\
\hline \multicolumn{6}{|l|}{ A. Relaciones } \\
\hline 1. Implicación (IM) & 4.18 & 2.53 & 4,53 & 2.20 & -05642 \\
\hline 2. Cohesión $(\mathrm{CO})$ & 3.56 & 1.89 & 3.71 & 2.03 & -0.2910 \\
\hline 3. Apoyo (AP) & 2.93 & 194 & 3.57 & 2.30 & -1.1554 \\
\hline \multicolumn{6}{|l|}{ B. Autorrealización } \\
\hline 4. Autonomia (AU) & 3.78 & 2.32 & 3.78 & 2.46 & -0.0072 \\
\hline 5. Organización $(\mathrm{OR})$ & 4.34 & 2.22 & 4.67 & 1.94 & -0.6166 \\
\hline 6. Presion (PR) & 4.15 & 1.60 & 5.10 & 1.54 & $-2.3248^{\circ}$ \\
\hline \multicolumn{6}{|l|}{ C. Estabilidad y Cambio } \\
\hline 7. Claridad (CL) & 4.00 & 2.65 & 3.75 & 2.31 & 0.3860 \\
\hline 8. Control $\{\mathrm{CN}\}$ & 4.78 & 199 & 478 & 2.06 & -0.0085 \\
\hline 9. Innovacion (IN) & 3.34 & 2.20 & 3.75 & 2.11 & -0.6763 \\
\hline 10.Comodidad (CF) & 4.21 & 2.18 & 421 & 2.28 & 0.0072 \\
\hline
\end{tabular}

*Significative al 0.05

CUADRO No 09-B: Comparación de las variables estrés laboral y percepción del clima social laboral en función a tiempo de servicio

Grupo $: A_{*}-$ Grupo *C*

\begin{tabular}{|c|c|c|c|c|c|}
\hline \multirow{2}{*}{ ÁREAS MHDHDAS } & \multicolumn{2}{|c|}{ Grupo ${ }^{+A}$ " } & \multicolumn{2}{|c|}{ Grupo "C" } & \multirow{2}{*}{ "t" } \\
\hline & $\mathbf{X}$ & D.S. & $\mathbf{X}$ & D.S. & \\
\hline \multicolumn{6}{|l|}{ L. Estrés laboral (burnout) } \\
\hline 1. Cansancio emocional (C.E) & 13.37 & 8.64 & 13.16 & 9.94 & 0.0872 \\
\hline 2. Despersonalización (Dp) & 653 & 4.89 & 5.4 & 4.90 & 0.8650 \\
\hline 3. Falta de Autorrealización personal ( $R p$ ) & 7.5 & 7.32 & 6.8 & 0.03 & 0.3862 \\
\hline \multicolumn{6}{|l|}{ II. Clima Social Laboral } \\
\hline \multicolumn{6}{|l|}{ A. Relaciones } \\
\hline 1. Imphcación (IM) & 4.18 & 2.53 & 5.2 & 2.41 & -1.5281 \\
\hline 2. Cohesion $(\mathrm{CO})$ & 3.56 & 1.89 & 3.64 & 1.89 & -0.1487 \\
\hline 3. Apoyo (AP) & 293 & 194 & 3.76 & 2.42 & -14215 \\
\hline \multicolumn{6}{|l|}{ B. Autorrealización } \\
\hline 4. Autonomia (AU) & 3.78 & 2.32 & 452 & 1.96 & -1.2736 \\
\hline 5. Organización (OR) & 4.34 & 2.22 & 4.92 & 2.28 & -09584 \\
\hline 6. Presión (PR) & 4.15 & 1.60 & 4.72 & 1.88 & -1.2184 \\
\hline \multicolumn{6}{|l|}{ C. Estabilidad y Cambio } \\
\hline 7. Claridad (CL) & 4.00 & 2.65 & 4.48 & 2.78 & -0.66 .33 \\
\hline 8. Control (CN) & 4.78 & 1.99 & 4.92 & 1.80 & -0.2717 \\
\hline 9 Innovación (IN) & 3.34 & 2.20 & 3.96 & 2.49 & -0.9882 \\
\hline 10.Comodidad (CF) & 4.21 & 2.18 & 3.28 & 1.92 & 1.5572 \\
\hline
\end{tabular}


CUADRO N 09-C: Comparación de las variables estrés laboral y percepción del clima social laboral en función a tiempo de servicio

Grupo *As - Grupo *Do

\begin{tabular}{|c|c|c|c|c|c|}
\hline \multirow{2}{*}{ ÁREAS MEDHAS } & \multicolumn{2}{|c|}{ Grupo "A" } & \multicolumn{2}{|c|}{ Grupo ${ }^{4} \mathrm{D} "$} & \multirow{2}{*}{ "t" } \\
\hline & $\mathbf{x}$ & D.S. & $\mathrm{x}$ & D.S. & \\
\hline \multicolumn{6}{|l|}{ I. Estrés laboral (burnout) } \\
\hline 1. Cansancio emocional (CE) & 13.37 & 8.64 & 15.75 & 14,34 & -0.6044 \\
\hline 2. Despersonalización (Dp) & 6.53 & 4.89 & 8.25 & 5.94 & -0.8521 \\
\hline 3. Falta de Autorrealización personal (Rp) & 7.5 & 7.32 & 13,37 & 7.20 & $-2.0355=$ \\
\hline \multicolumn{6}{|l|}{ II. Clima Social Laboral } \\
\hline \multicolumn{6}{|l|}{ A. Relaciones } \\
\hline 1. Implicación (IM) & 4.18 & 2.53 & 4.75 & 320 & 0.5295 \\
\hline 2. Cohesion $(\mathrm{CO})$ & 3.56 & 1.89 & 3,37 & 1.59 & 0.2455 \\
\hline 3. Apoyo (AP) & 2.93 & 1.94 & 3.12 & 2.29 & -0.2350 \\
\hline \multicolumn{6}{|l|}{ B. Autorrealización } \\
\hline 4. Autonomia (AU) & 3.78 & 2.32 & 4.0 & 2.13 & -0.2415 \\
\hline 5. Organización (OR) & 4.34 & 2.22 & 387 & 2.35 & 0.5274 \\
\hline 6. Presión (PR) & 4.15 & 1.60 & 3.5 & 1.80 & 1.0326 \\
\hline \multicolumn{6}{|l|}{ C. Estabilidad y Cambio } \\
\hline 7. Claridad (CL) & 4,00 & 2.65 & 5.0 & 3.02 & 0.9286 \\
\hline 8. Control $(\mathrm{CN})$ & 4.78 & 1.99 & 4.37 & 1.68 & 0.5291 \\
\hline 9. Innovalición (IN) & 3.34 & 2.20 & 2.75 & 1.76 & 0.7045 \\
\hline 10.Comodidad (CF) & 4.21 & 2.18 & 3.0 & 2.20 & 1.2657 \\
\hline
\end{tabular}

+ Significativo al 0.05

CUADRO N*09-D: Comparación de las variables estrés laboral y percepción del clima social laboral en función a tiempo de servicio Grupo $\mathrm{B}^{\mathrm{B}}-\mathrm{Grupo} * \mathrm{C}$.

\begin{tabular}{|c|c|c|c|c|c|}
\hline \multirow{2}{*}{ ÁREAS MEDIDAS } & \multicolumn{2}{|c|}{ Grupo "B" } & \multicolumn{2}{|c|}{ Grupo "C" } & \multirow{2}{*}{ "t" } \\
\hline & $\mathbf{x}$ & D.S. & $\mathbf{x}$ & D.S. & \\
\hline \multicolumn{6}{|l|}{ I. Estrés laboral (burnout) } \\
\hline 1. Cansancio emocional (CE.) & 16.96 & 10.13 & 13.16 & 9.94 & 1.3763 \\
\hline 2. Despersoralización (Dp) & 7.57 & 4.78 & 5.4 & 4.90 & 1.6289 \\
\hline 3. Falta de Autorrealización personal ( $\mathrm{Rp}$ ) & 6.92 & 8.25 & 6.8 & 0.03 & 0.0759 \\
\hline \multicolumn{6}{|l|}{ II. Clima Social Laboral } \\
\hline \multicolumn{6}{|l|}{ A. Relaciones } \\
\hline 1. Implicación (IM) & 4.53 & 2.20 & 52 & 2.41 & -1.0473 \\
\hline 2. Cohesión (CO) & 3.71 & 2.03 & 3.64 & 1.89 & 0.1371 \\
\hline 3. Apoyo (AP) & $3.5 ?$ & 2.30 & 3.76 & 2.42 & 402907 \\
\hline \multicolumn{6}{|l|}{ B. Autorrealización } \\
\hline 4. Autonomfa (AU) & 3.78 & 2.46 & 4.52 & 1.96 & -1.1888 \\
\hline 5. Organización (OR) & 4.67 & 1.94 & 4.92 & 2.28 & -0.4150 \\
\hline 6. Presión (PR) & 5.10 & 1.54 & 4.32 & 1.88 & 0.8211 \\
\hline \multicolumn{6}{|l|}{ C. Estabilidad y Cambio } \\
\hline 7. Claridad (CL) & 3.75 & 2.31 & 4.48 & 2.78 & -1.0405 \\
\hline 8. Control (CN) & 4.78 & 2.06 & 4.92 & 1.80 & -0.2511 \\
\hline 9. Innovación (IN) & 3.75 & 2.11 & 3.96 & 2.49 & -0.3094 \\
\hline 10.Comodidad (CF) & 4.21 & 2.28 & 3.28 & 192 & 1.5997 \\
\hline
\end{tabular}


CUADRO N ${ }^{\circ}$ 09-E: Comparación de las variables estrés laboral y percepción del clima social laboral en función a tiempo de servicio

Grupo *B* - Grupo *D*

\begin{tabular}{|c|c|c|c|c|c|}
\hline \multirow{2}{*}{ ÁREAS MEDIDAS } & \multicolumn{2}{|c|}{ Grupo "B" } & \multicolumn{2}{|c|}{ Grupe ${ }^{*} D^{n}$} & \multirow{2}{*}{$" t$} \\
\hline & $\mathrm{x}$ & D.S. & $\mathbf{x}$ & D.S. & \\
\hline \multicolumn{6}{|l|}{ 1. Estrés laboral (burnout) } \\
\hline 1. Cansancio emocional (C.E.) & 16.96 & 10.13 & 15.75 & 14.34 & 0.2721 \\
\hline 2. Despersonalización (Dp) & 7.57 & 4.78 & 8.25 & 5.94 & -0.3353 \\
\hline 3. Falta de Autorrealización personal (Rp) & 6.92 & 8.25 & 13.37 & 7.20 & $-2.4866^{\circ}$ \\
\hline \multicolumn{6}{|l|}{ II. Clima Social Laboral } \\
\hline \multicolumn{6}{|l|}{ A. Relaciones } \\
\hline 1. Implicación (IM) & 4.53 & 2.20 & 4.75 & 3.20 & -0.2169 \\
\hline 2. Cohesión $(\mathrm{CO})$ & 3.71 & 2.03 & 3.37 & 1.59 & 0.4335 \\
\hline 3. Apoyo (AP) & 357 & 230 & 3.12 & 2.29 & 0.4843 \\
\hline \multicolumn{6}{|l|}{ B. Autorreatización } \\
\hline 4. Autonomía (AU) & 3.78 & 2.46 & 4.0 & 2.13 & -0.2222 \\
\hline 5. Organización (OR) & 4.67 & 1.94 & 387 & 235 & 0.9843 \\
\hline 6. Presión (PR) & 5.10 & 154 & 3.5 & 1.80 & $2.5708^{*}$ \\
\hline \multicolumn{6}{|l|}{ C. Estabilidad y Cambio } \\
\hline 7. Claridad (CL) & 3.75 & 2.31 & 5.0 & 3.02 & -1.2568 \\
\hline 8. Control (CN) & 4.78 & 2.06 & 4.37 & 1.68 & 0.5148 \\
\hline 9. Innovación (IN) & 3.75 & 2.11 & 2.75 & 1.76 & 1.0759 \\
\hline 10 Comodidad (CF) & 4.21 & 2.28 & 3.0 & 2.20 & 13361 \\
\hline
\end{tabular}

- Significativo al 0.05

CUADRO N 09-F: Comparación de las variables estrés laboral y percepción del clima social laboral en función a tiempo de servicio Grupo *C" - Grupo *D*

\begin{tabular}{|c|c|c|c|c|c|}
\hline \multirow{2}{*}{ AREAS MEDIDAS } & \multicolumn{2}{|c|}{ Grupo $" \mathrm{C} " \mathrm{C}$} & \multicolumn{2}{|c|}{ Grupo "D" } & \multirow{2}{*}{$" t "$} \\
\hline & $\mathbf{X}$ & D.S. & $\mathbf{x}$ & D.S. & \\
\hline \multicolumn{6}{|l|}{ I. Estrés laboral (burnout) } \\
\hline 1. Cansancio emocional (C.E.) & 13,16 & 9.94 & 15.75 & 14.34 & -0.5747 \\
\hline 2. Despersonalización (Dp) & 5.4 & 4.90 & 8.25 & 5.94 & -1.3596 \\
\hline 3. Falta de Autorrealización personal (Rp) & 6.8 & 0.03 & 13.37 & 7.20 & $-2.5615^{\circ}$ \\
\hline \multicolumn{6}{|l|}{ II. Clima Social Laboral } \\
\hline \multicolumn{6}{|l|}{ A. Relaciones } \\
\hline 1. Implicación (IM) & 5.2 & 241 & 4.75 & 320 & 0.4201 \\
\hline 2. Cohesión (CO) & 3.64 & 1.89 & 3.37 & 1.59 & 0.3568 \\
\hline 3. Apoyo (AP) & 3.76 & 2.42 & 3.12 & 2.29 & 0.6534 \\
\hline \multicolumn{6}{|l|}{ B. Autorrealización } \\
\hline 4. Autonomia (AU) & 452 & 1.96 & 4.0 & 2.13 & 0.6394 \\
\hline 5. Organización (OR) & 4.92 & 228 & 3.87 & 2.35 & 1.1160 \\
\hline 6. Presión (PR) & 4.72 & 1.88 & 3.5 & 1.80 & 1.6473 \\
\hline \multicolumn{6}{|l|}{ C. Estabilidad y Cambio } \\
\hline 7. Claridad (CL) & 4.48 & 2.78 & 5.0 & 3.02 & -0.4505 \\
\hline 8. Control $(\mathrm{CN})$ & 4.92 & 1.80 & 4.37 & 1.68 & 0.7557 \\
\hline 9. Innovación (IN) & 3.96 & 2.49 & 2.75 & 1.76 & 1.2703 \\
\hline 10.Comodidad (CF) & 328 & 192 & 3.0 & 2.20 & 03459 \\
\hline
\end{tabular}

- Significative al 0.05 
B. Análisis factorial de la prueba de clíma social laboral (Cuadro $\mathrm{N}^{\circ} 10-\mathrm{A}$ y 10-B) A pesar de que no es motivo de la investigación, se ha creído conveniente realizar el análisis factorial de la prueba Clima Social Laboral en la medida que no existen experiencias real izadas en nuestro medio, sin embargo, en la prueba de estrés laboral (Burnout) sí se han realizado.

Los resultados nos indican que las diez subescalas que consta la prueba pueden ser explicadas en base a dos componentes:

- Componente 01: Conformado por las subescalas Apoyo (AP), Autonomía (AU), Comodidad (CF), Claridad (CU, Cohesión (CO), Implicación (1M), Innovación (IN) y Organización (OR).

- Componente 02: Conformado por las subescalas Control (CN) y Presión (PR).

CUADRO $\mathrm{N}^{\circ}$ 10-A: Matriz de componentes

\begin{tabular}{ccc}
\hline & Comp. 1 & Comp. 2 \\
\hline AP2 & 0.79 & -0.17 \\
AU2 & 0.78 & -0.28 \\
CF2 & 0.58 & 0.07 \\
CL2 & 0.88 & -0.03 \\
CN2 & 0.26 & 0.79 \\
C02 & 0.84 & -0.04 \\
IM2 & 0.85 & 0.23 \\
IN2 & 0.79 & -0.14 \\
OR2 & 0.76 & 0.31 \\
PR2 & -0.26 & 0.56
\end{tabular}

CUADRO $\mathrm{N}^{\circ}$ 10-B: Matriz de componentes rotados

\begin{tabular}{ccc}
\hline & Comp. 1 & Comp. 2 \\
\hline AP2 & 0.79 & -0.17 \\
AU2 & 0.78 & -0.27 \\
CF2 & 0.58 & 0.07 \\
CL2 & 0.88 & -0.03 \\
CN2 & 0.26 & 0.79 \\
C02 & 0.84 & -0.04 \\
IM2 & 0.85 & 0.23 \\
IN2 & 0.79 & -0.14 \\
OR2 & 0.76 & 0.31 \\
PR2 & -0.26 & $\mathbf{0 . 5 6}$ \\
& & \\
\hline
\end{tabular}




\section{CONCLUSIONES}

1. No existe correlación entre las escalas de estrés laboral y la escala de clima social laboral en la muestra estudiada.

2. Entre los profesionales contratados y nombrados solo existe diferencia en la sub prueba de Presión (PR) de la escala de clima social laboral.

3. No se encuentra diferencia significativa en ambas variables en la muestra en función a género.

4. Los profesionales de la salud, Médicos, Enfermeras y Tecnólogos Médicos son los que presentan una mayor disposición, como consecuencia de su actividad profesional, de presentar un mayor nivel de estrés o agotamiento profesional, estableciéndose por tal motivo diferencia significativa en relación a los otros profesionales de la salud: Obstetriz, Psicólogo, Trabajo Social.

5. No se encuentran diferencias en la escala de clima social laboral entre los profesionales de la salud, salvo en el caso de los tecnólogos médicos, cuya percepción es desfavorable, en el área de Relaciones.

6. En lo que respecta al tiempo de servicio, el Grupo O, constituye el grupo crítico, ya que el nivel de estrés laboral experimentado por ellos es mayor en función a los otros grupos de comparación, con manifestaciones de desmotivación y descontento interior.

7. El grupo B percibe una mayor presión de parte de la Institución hospitalaria en función a los otros grupos de comparación.

8. Desde el punto de vista del análisis factorial, las diez subescalas de la prueba de Clima Social Laboral puede agruparse en dos componentes básicos. 


\section{REFERENCIAS}

1. Moos, R.H.; TrickeU, E.J. (1984) Escala de clima social. TEA, Madrid

2. Delgado, A. (2002) Sindrome del Burnout en profesores de educación secundaria de Lima Metropolitana. Revista del Instituto de Investigaciones Psicológicas. UNMSM. Vol. 6 No 2

3. Ponce, C. (2001) Sindrome del Burnout en docentes universitarios. XXIX Congreso Interamericano de Psicología. Julio 2003 Lima-Perú

4. Pulido, C (2002) Construcción, validación y aplicaciones de Una prueba que mide el clima en organizaciones empresariales privadas y su implicación para la psicología de la salud. Tesis para optar el grado de Magíster en Psicología. UNMSM.

5. Weinert, A. (1985) Manual de Psicología de la organización: La conducta humana en las organizaciones. Barcelona: Harder

6. Kolb, D.; Rubin 1.; Mclntyre, J. (1977) Psicología de las organizaciones. México; 1 ra Edición, Prentice Hall.

7. Gil-Monte, P. y Peiró, J. (1997). Desgaste psíquico en el trabajo: síndrome de quemarse. Madrid: Editorial Síntesis SA

8. Brunet, L.(1987) El clima de trabajo en las organizaciones. Definición, diagnóstico y consecuencias. México:

Editorial Trillas.

9. Maslach, C. y Jackson, S. (1997).

Inventario «Burnout» de Maslach. Madrid: Editorial TEA. 